\title{
Affect on Several Control Strategies of A Model for Malaria in an Endemic Region Like Bangladesh
}

\author{
M. M. Islam, M. R. Islam* and M. M. Rahman \\ Mathematics Discipline, Khulna University, Khulna-9208, Bangladesh,
}

\begin{abstract}

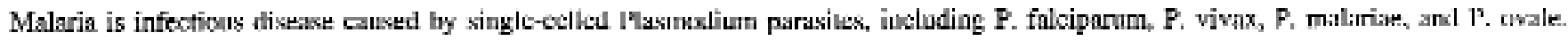
The parasitcs are asunlly trunsmilled from infected to non infecled people via ule bitc of fenale Anopbeles mosquitoes about 60 .precies af Arropheles can scrve as vectors. We hive seen that milnria is endemic in develuring countrics like Bangladesh where inadequate drninage provites water logeing which are the suitnble breeding sectors of the disense veclur. In this puper a sinule SiS inodel is formulatod

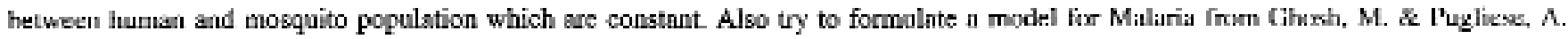

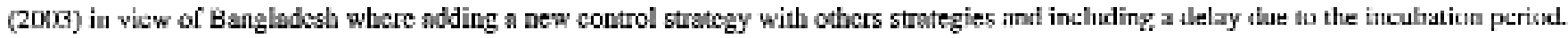
llere diwuswed several controls of stratcgics on the transmission of utalaria. The control strategies incrupurated here were: (i) bicklcyical

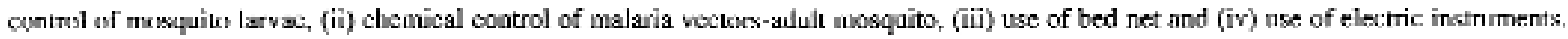
This motel ix tried in analyne by diffurcutial cqualions, buclude prow of a thoorem and compater imitation. Various control strategies

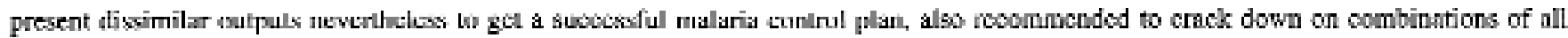
control strategies,
\end{abstract}

Key words: Mathematicnl modeling, Endemic region and SIS Model

\section{Introduction}

Malarta is an acute fln-like illness causod hy one of four species of parasile of the genus Plasmodium (Discussed above). The malaria disease is most commonly transmitted to humans through a bite of an infected femule Anopheles unosuuitó. Malaria is most common in tropical and sublropical lands, particularly (More than $80 \%$ cases) in Africa and south Asia: Bungladesh, India, Sri Lanka and ctc. Also malaria is a curahle discase if promptly and adequately treated. When an Anopheles mosquito ingests blowd from a malaria-infected person, malaria parasites develop in the mosquito and migrate into the mosquito's salivary glands. While rare, the malaria parksite can also be transmilled by transfusion with infected blood, by shared needle use, or from a mother to her unhom child. By Malaria about 300-500 milliva peoples are affected in the world and kill more than 1.5 million Peuple annually. Fur detwils we can be seen WHO (world mularia report 2005). 'There are various control methods to maintain the spread of malaria implemented earlier in different parts of the world. Though every control strategy is not valid for every region, there are neveral other factors which in luence discase transmission and also the control measurce. For details one can scc Walker (1992). Now few control meisures alre distussed:

\section{(i) Biolongical control of musquilo larvae}

The larval is the first stape of mosquitocs which can he controlled by biologically. The principal biological coninol agents that have been successfully empluyed ingainst Anophelcs are predators, particularly fish and the some bacterial pathogen that ittack the larval stage of the mosquito. For detail we cun the seen Das, P.K., Amalraj. D.D. (1997).

\section{(ii) Chemical control of malaria vectors adult mosquito}

Chemical control of andult femile muscivito has beent the most flourishing vector control methods since 1y40. 'The nosi common practice is indoor residual house spraying of insecticides. DDT is the rnost widely usexl insecticide for vcctor control. l'or a long time the people in endentic region usc mosquito coil as in housc control strategies.

\section{(iii) Ust of bod ntt}

Mosquitues which are stait biting scrionsly after smnect. Actually they bite in any dark plase. In $\Lambda$ frical, the maliaria

*Corresponding author, E-mạil: : smrislam_66@yahuo.coms: 
purusite accounts for at least $\mathbf{2 5 \%}$ of all childhood mortality helow age live. Bangladesh is one of the risky plictes for breading mosquitoes hocause of our environment. By using bed net poople can save them from malaria. Bed net is also ideal becanse it protect environment from uny pollution.

\section{(iv) Use of electric instruments}

At the present time we huve used different types of electric instrument which can control wdult mosquitocs such as Electric net, Megu-calch, Mat which ure used only at night. It is pussible to die more then 1200 mosquito at u lime by using Mega-calch. Now a day this control strategy is very eflective all over the world. So hy using this method we can control mosquitocs hroeding.

In Africa, southern part of Asia like India, Bangladesh, SriLunka and etc persons who have heen repeatedly infecited by malaria a degree of immunity which suppresses most clinical symptoms. The people carry gurnetocytes in their blood that infect the mosquilves biting them and form al separatc class of reservoir population which helps in spreading malaria without getting affected thernselves. For detail we can he scen Despummier, D.D., Gwadz, R.W., Hotez, P.J. (1994) \& Nchinda, T.C. (1998). In this paper two models are coustructed including this class of human popululion. Although there have been scveral experimentil studics related to surveys of maluria in different rcgions. Dus, S.C., Bhuyan, M., Baruah, T.., Talukder, P.K. (1991) \& Sharma, V.P. (1996, 1998) they discussed, spread of discasc using mathematicul mudels by considering diflerent control stralegies and delays due to the incuhation period has not been conducted, particularly when the densitics of the human and mosquito populations are variable. Here cach control strattegy is nol valid for cach region.

\section{A Simple SIS Model}

we take only susceptible and infective classes from the two species (Homan \& mosquitoes). This model is based on criss-cross interaction between this two species. I ct, human and female mosquilues both are constants which are shown in figure. For detail we can be seen Bailcy, N.T J. (1975). I.ct the total number of human and musquitoes are $H$ and $M$ respectively. 'Then

$$
\begin{aligned}
& S_{H}(t)+I_{M}(t)=H \\
& S_{\text {J }}(t)+I_{M}(t)=M
\end{aligned}
$$

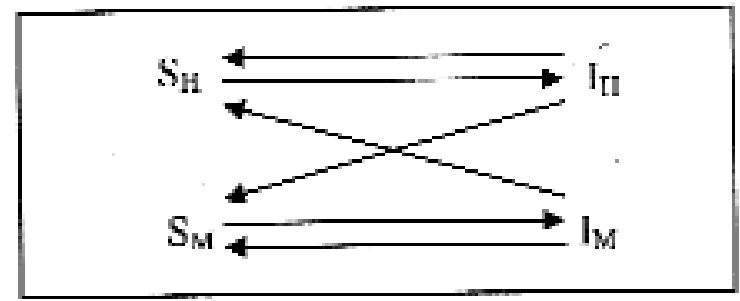

linst we consider, the ralte of decrcase of human susceptible $\left(S_{H}\right)$ to be proportional to the human susceptible tinues the inlectious mosquito $\left(I_{M}\right)$ with a similar furm for the. mosquito rate. In this sase, if some how any one infective. human $\left(I_{H}\right)$ or mosquilues can he rocoverwd, then they rejoin the susceptible class. Theo the model (2.1.1) becoune,

$$
\begin{aligned}
& \frac{d S_{H}}{d t}-r_{1} S_{U} I_{M}+\alpha_{1} I_{H} \\
& \& \frac{d I_{U}}{d t}=r_{1} S_{H} I_{M}-\alpha_{1} I_{H} \\
& \frac{d S_{M}}{d t}=-r_{2} S_{M} I_{H}+\alpha_{2} I_{H} \\
& \& \frac{d I_{M}}{d t}=r_{2} S_{M} I_{H}-\alpha_{2} I_{H}
\end{aligned}
$$

where $r_{1}, \alpha_{1}, r_{2}$ and $\alpha_{2}$ are positive parameters. At the initial condition we consider,

$S_{H}(0)=S_{H_{a}} \& S_{M}(0)=S_{M_{u}}$

$I_{H}(0)=I_{U_{s}} \& \quad I_{G}(0)=I_{M_{*}}$

But (2.1.3) is contain four cquations i.e.

$4^{\text {th }}$ order system with (2.1.2) it reduces to a second order system in either $S_{H}$ and $S_{M}$ or $I_{n}$ and $I_{w}$. Then

$\frac{d I_{H}}{d t}-r_{1}\left(I I-I_{M}\right) I_{M}-\alpha_{1} I_{U}$
$\frac{d I_{M}}{d t}=r_{3}\left(M-I_{M}\right) I_{H}-\alpha_{2} I_{M}$

I ct, the equilibrium points $\left(I_{H}, I_{M}\right)$ duat are stcady states of $(2.1 .4)$ are $I_{H}=0=I_{M}$. Then

$\frac{d I_{H}}{d t}=0 \& \frac{d I_{M}}{d t}=0$

From (2.1.5),

$I_{B}=\frac{I_{M} H}{I_{M}+P_{1}} \quad\left[\because P_{1}=\frac{\alpha_{1}}{r_{1}}\right]$

and, $I_{M}=\frac{I_{I I} M}{I_{H}+P_{2}}\left[\because P_{2}=\frac{\alpha_{2}}{r_{2}}\right]$

From (2.1.6) and (2.1.7) 


$$
\begin{aligned}
& M I_{H}{ }^{2}+P_{1} I_{H}{ }^{2}+P_{1} P_{2} I_{u}=H M I_{u} \\
& \therefore I_{H}=0 \text { and } I_{H}=\frac{H M-P_{1} P_{2}}{M+P_{1}}
\end{aligned}
$$

Similarly,

$$
I_{M}=0 \text { and } I_{M}=\frac{H M-P_{1} P_{2}}{H+P_{2}}
$$

Therefore, non-7cro positive steady state levels of the infective population exist only if $\frac{H M}{P_{1} P_{2}}>1$, which is the threshold condition; if the positive stcady state cxists then the zero steady state is unstable. This is indeed the case. In metric form as a lincarization of (2.1.5)

$$
\frac{d}{d t}\left(\begin{array}{l}
I_{U} \\
I_{M}
\end{array}\right)=\left(\begin{array}{cc}
-\alpha_{1} & r_{1} H \\
r_{2} M & -\alpha_{2}
\end{array}\right)\left(\begin{array}{l}
I_{H} \\
I_{M}
\end{array}\right)
$$

(Here, neglected non-linear term). Now find the Eigen values for the lincarization

of (2.1.5) about $I_{H}=0=I_{M}$ are given by

$$
\begin{aligned}
& \left(\begin{array}{cc}
-\alpha_{1} & r_{1} H \\
r_{2} M & -\alpha_{2}
\end{array}\right)-\left(\begin{array}{ll}
\lambda & 0 \\
0 & \lambda
\end{array}\right)=0 \\
& \therefore \lambda=-\frac{-\left(\alpha_{1}+\alpha_{2}\right) \pm \sqrt{\frac{\left(\alpha_{1}+\alpha_{2}\right)^{2}+}{4 \alpha_{1} \alpha_{2}\left(\begin{array}{l}
H M \\
P_{1} P_{2}
\end{array}-1\right)}}}{2}
\end{aligned}
$$

Here, if the threshold condition $\frac{H M}{P_{1} P_{2}}>1$ holds and $\lambda_{1}<0<\lambda_{2}$ then the origin $(0,0)$ is u saddle point in the phase plunc $\left(I_{H}, I_{M}\right)$. If the threshold condition is not salisfied that is $\frac{I L M}{P_{1} P_{2}}<1$ then the origin is stahlc since both $\lambda<0$. In this case $I_{M}$ is pusitive and $I_{M}$ does not exist. Again if $I_{n}$ and $I_{s}$ exist, meaning in the context here that they are positive, then linearization (2.1.5) about it, if the Eigen valucs $\lambda$ satisfy. In metric form of (2.1.5)

$$
\frac{d}{d t}\left(\begin{array}{l}
I_{I} \\
I_{M}
\end{array}\right)=\left(\begin{array}{cc}
-\alpha_{1}-r_{1} I_{M} & r_{1} H-r_{1} I_{H} \\
r_{2} M-r_{2} I_{M} & -\alpha_{2}-r_{2} I_{U}
\end{array}\right)\left(\begin{array}{l}
I_{I} \\
I_{M}
\end{array}\right)
$$

For, Ligen values

$$
\begin{aligned}
& \lambda^{2}+\lambda\left(\alpha_{1}+\alpha_{2}+r_{1} I_{M}+r_{2} I_{n}\right)+ \\
& {\left[\alpha_{2} r_{1} I_{M}+\alpha_{1} r_{2} I_{H}+\alpha_{1} \alpha_{2}\left(I_{M} H+I_{M} M\right)+\right.} \\
& \left.\alpha_{1} \alpha_{2}-r_{1} r_{2} H M\right]=0
\end{aligned}
$$

is a characteristic equation where $\operatorname{Re}(\lambda)<0$ and the positive steady state $\left(l_{n}, l_{M}\right)$ is stible. So, the threshold condition for a non-7ero stertly slate infected population is $\frac{H M}{P_{1} P_{1}}>1$. Now if cvery human is susceptible, then $\frac{r_{1} H}{\alpha_{1}}$ is the average number of human cuntakled by a mosquits infeclive during its infectious period and visc versa for $\frac{r, M}{\alpha_{2}}$. Now $\frac{r_{1} H}{\alpha_{1}}$ and $\frac{r_{2} M}{\alpha_{2}}$ are the maximal human and mosquito contact rates respectively. 'This is not realistic for long term study of spreud of discasc. Thes in following article, a movel is formulatcd where humin und mosquito population is variable and including diffecent control stratcgics.

\section{The Mathematical Model}

We consider, only two stages (larvae and idult) ol mosquito population ure considened in this article us it stays in pupa stage unly for 4 days. So it is hetter to incluble delay in mosquito from lurval stage to adult slage. liemale mosquitoes of Anopheles species are the main culprit for breeding malaria in human population. So we lake valy female masquitues for this model, For detisils we cun be seen Ghush, M. \& Pugliese, $\Lambda$. (2003). Suppose a fraction $f$ of total mosquito density $(D)$ is female then $f D-D_{\text {io }}$ (the density of [enale mosquitocs). The growtla equaltiuns for the density of mosquito larvae $(L)$ and for the density of adult musquito $(D)$ assuming density dependent monality for both stages can be written as follows: (Here vee saln put some parameters zero to exccute spocific control methods suitable to (hatl region).

$$
\dot{L}=b f D-\left(\lambda+\lambda_{1} L\right) L-c F(t) L
$$

$\dot{D}=c F(t) L-\left(\mu+\mu_{1} D\right) D$

Wherc, $b=$ is the population rate per individual, $c=$ is the scttlement rate per unit of free space, $\int=\mathrm{a}$ [ration of tolal mosquito density is female, $\lambda=$ per capital death rales in 
the larval of mosyuito, $\lambda_{1}=$ is constants corresponding to density dependent deaths in larvae . $\mu=$ per capital death rates in adult stages of mosquilo, $\mu_{1}=$ is constants corresponding to density dependent death in adult population(Breeding spacc),$F(t)=$ is the frec spuce al lime L. Frum (3.1.1) we get

$$
\begin{aligned}
& \dot{L}=b D_{n}-\left(\lambda+\lambda_{1} L\right) L-c F(t) L \\
& \dot{D}_{s}=f c F(t) L-\left(\mu+\mu_{1} D_{n}\right) D_{n}
\end{aligned}
$$

we consider, female musquito into two classes such as susceptible class $\left(X_{-}\right)$aAd infective class $\left(Y_{n}\right)$. Now we are going to model the spread of malaria in epidemic region. Here the total human population density $D_{h}$ into three classes such as snisceptible class $\left(X_{b}\right)$, infective class $\left(Y_{b}\right)$. reservoir class $\left(Z_{k}\right)$.Then the Model for the spread of malaria can be written, for details (ihosh, M. (2000)).

$$
\begin{aligned}
& \dot{X}_{k}=\left(b_{k}-f \frac{r_{k}}{k} D_{k}\right) D_{h}- \\
& {\left[d_{\mathrm{s}}+(1-f) \frac{r_{\mathrm{a}}}{K} D_{\mathrm{s}}\right] X_{\mathrm{a}}-\beta X_{\mathrm{si}} Y_{\mathrm{s}}+v Y_{k}} \\
& \dot{Y}_{k}=\beta X_{b} Y_{s m}-\left[\begin{array}{l}
v+\eta+d_{k}+\delta+ \\
(1-f) \frac{r_{b}}{K} D_{b}
\end{array}\right] Y_{b}, \\
& \dot{Z}_{k}=\delta Y_{\underline{\underline{\underline{s}}}}-\left\{d_{\mathrm{a}}+(1-f) \frac{r_{\underline{\underline{b}}}}{K} D_{\mathrm{n}}\right\} Z_{\mathrm{a}} \text {, } \\
& \dot{D}_{b}=r_{h}\left(1-\frac{D_{h}}{K}\right)-\eta Y_{k} \text {, } \\
& \dot{X}_{m}=f c F L-\left(\mu+\mu_{1} D_{n}\right) X_{n}- \\
& \beta X_{m} Y_{\text {is }}-\gamma X_{m} Z_{k} \text {, } \\
& \dot{Y}_{\mathrm{m}}=\beta^{\prime} X_{m} Y_{k}+\gamma Y_{s} Z_{\Delta}-\left(\mu+\mu_{1} D_{n}\right) Y_{\mathrm{at}} . \\
& \dot{D}_{w}=f c F L-\left(\mu+\mu_{1} D_{w}\right) D_{w}
\end{aligned}
$$

where, $b_{b}$ and $d_{s}$ are natural birth rate $\&$ death rate respectively, $r_{\mathrm{a}}=b_{\mathrm{a}}-d_{\mathrm{a}}$ is the growth rate, $K=$ is the carrying cupucity of the huminn population in the natural cnvironment, where $0<f<1$ is the convex combination constant which governs the logistic hirth and logistic death of the humban population, when $f=1$, then the model is logistic birth model as all of the restricted growth is due to decreusing birth rate and the death rate is constant, When $f=0$ the model is logistic death mode] als all of the restricted growth is due to an increasing death rate and birth rate is constant (Gas, L.Q. Hetheote, H.W. 1992), $v=$ is the recovery rale constant, $\eta=$ is the disease related death rates constanh, $\beta-$ is the interiction coefficient of the susceptihle human with the infective mosquito population, $\beta^{\prime}=$ is the interaction coefficient of susceptible mosquito with the inlective buman ctass, $\gamma=$ is the inleraction coefficient of susceptible mosquito wilh the humun reservoir class, $\delta=$ is the cocfficient corresponding to the movement of human from infective elass to reservoir class.

\section{The Mathematical model with controls and delays}

T.et, $K_{1}, \kappa_{2}, K_{3}$ are the constants corresponding to the death of larvae due to the biological control, the chemical control, the death of adult mosquito due the electric instinument respectively. $f_{p p}=$ is the fraction of total population which uses hed net. Thus the fraction $\left(1-\int_{10}\right)$ of eath thass of bunan are exposed to mosquito bites. $\Lambda$ gain we consider, $\tau_{1}$ and $\tau_{2}$ are the delay due to the incuhation period in human \& moscuito respectively and $\tau=$ is the delay comesponding to survival from tarvae to adult stinges. Thus the Model hecanc:

$$
\begin{aligned}
& \bar{L}=b D_{\mathrm{m}}-c F L-\left(\lambda+\lambda_{1} L\right) L-\kappa_{1} L \\
& \dot{Y}_{a}=\beta^{\prime}(1-f)\left(D_{m}-Y_{m}\right)\left(t-\tau_{1}\right) Y_{k}\left(t-\tau_{1}\right)+ \\
& \gamma\left(1-f_{k p}\right) Z_{h}\left(t-\tau_{1}\right)\left(D_{m}-Y_{n}\right)\left(t-\tau_{l}\right)- \\
& \left(\mu+\mu_{1} D_{m}\right) Y_{m}-\kappa_{2} Y_{m}-\kappa_{1} Y_{s} \\
& \dot{D}_{\mathrm{w}}=f c F L(t-\tau)-\left(\mu+\mu_{1} D_{\mathrm{m}}\right) D_{\mathrm{m}}- \\
& \mathrm{K}_{2} D_{\mathrm{s}}-\mathrm{\kappa}_{3} D_{\mathrm{n}} \\
& \bar{Y}_{h}=\beta\left(1-f_{i j}\right)\left(D_{h}-Y_{k}-Z_{k}\right)\left(t-\tau_{2}\right) \\
& Y_{m}\left(I-\tau_{2}\right)-\left(v+\eta+d_{k}+\delta+(I-f) \frac{r_{h}}{K}, D_{k}\right) Y_{k} \\
& \dot{Z}_{\mathrm{h}}=\delta Y_{\mathrm{s}}-\left\{d_{k}+(1-f) \frac{r_{\text {至}}}{K} \cdot D_{h}\right\} Z_{\mathrm{s}} \\
& \& D_{h}=r_{b}\left(1-\frac{D_{h}}{K}\right) D_{h}-\eta Y_{\underline{b}}
\end{aligned}
$$


Here, the inilial condition is $L(t)=I_{0}(t) \geq 0$, where $L_{0}(t)$ is given non ncgative function on $-\tau \leq t \leq 0$, $D_{i}(t)=D_{k 0}(t) \geq 0$, where $D_{b 0}(t)$ is given rion-ncgative function on $-\tau_{1} \leq t \leq 0, Y_{h}(t)=Y_{\mathrm{a} 0}(t) \geq 0$, where $Y_{\mathrm{h} 0}(t)$ is given non-negative function on $-\tau_{1} \leq t \leq 0$, $Z_{k}(t)=Z_{n 0}(t) \geq 0$, where $Z_{n 0}(t)$ is given nonnegative function on $-t_{1} \leq t \leq 0, \quad Y_{m}(t)=Y_{m}(t) \geq 0$, where $Y_{m 0}(t)$ is given nonnegative function on $-\tau_{2} \leq t \leq 0$. $D_{m}(t)=D_{m 0}(t) \geq 0$, where $D_{n \theta}(t)$ is given nonnegative function on $-t_{2} \leq t \leq 0$, The steady state equilibrium of the system (3.2.1) can be derived als follows froun the first and third equation we get, from first cquation, $i=0$

$\therefore b D_{m}-c F L-\left(\lambda+\lambda_{1} L\right) L-\kappa_{1} L=0$

From third equation of $(3.2 .1), \bar{D}=0$;

$\Rightarrow L f c F-\left(\mu+\mu_{1} D_{m}\right) D_{m}-\kappa_{2} D_{m}-\kappa_{3} D_{m}=0$

$\therefore L=\frac{\left(\mu+\mu_{1} D_{m}\right) D_{m} K_{2} D_{m}+\kappa_{3} D_{m}}{f c F}$

From (3.2.3) \& (3.2.2),

$D_{m}\left\{b-\left(c F+\lambda+\kappa_{1}\right) \frac{\left(\mu+\mu_{1} D_{\mathrm{n}}+\kappa_{2}+\kappa_{1}\right)}{f c F}-\right.$
$\left.\lambda_{2}\left(\frac{\mu+\mu_{1} D_{n}+\kappa_{2}+\kappa_{3}}{f c F}\right)^{2} D_{n}\right\}=0$

Thus $D_{m}=0$ and

$$
\begin{aligned}
& \lambda_{1}\left(\frac{\mu_{1}}{\mu c F}\right)^{2} D_{w}^{3}+\frac{2\left(\mu+\kappa_{2}+\kappa_{3}\right) \mu_{1} \lambda_{1}}{(f c F)^{2}} D_{w}{ }^{2} \\
& \left\{\left(\frac{\mu+\kappa_{2}+\kappa_{3}}{f c F}\right)^{2}+\left(\frac{c F+\lambda+\kappa_{1}}{f c F}\right) \mu_{1}\right\} D_{w}+ \\
& \left(\frac{c F+\lambda+\kappa_{1}}{f c F}\right)\left(\mu+\kappa_{2}+\kappa_{3}\right)-b=0
\end{aligned}
$$

where all the cocfficients are positive except the constant term. For existence of unique positive root (say $D_{m}^{*}$ ) of (3.2.5), we nood the following condition:

$$
\begin{aligned}
& \left(\frac{\lambda+\kappa_{1}+c F}{f c F}\right)\left(\mu+\kappa_{2}+\kappa_{3}\right)-b>0 ; \\
& \therefore F>\frac{\left(\lambda+\kappa_{1}\right)\left(\mu+\kappa_{2}+\kappa_{3}\right)}{\left\{f b-\left(\mu+\kappa_{2}+\kappa_{3}\right)\right\} c}
\end{aligned}
$$

At the stesidy state,

$$
\begin{aligned}
& \dot{D}_{\hat{L}}=r_{h}\left(1-\frac{D_{k}}{K}\right) D_{k}-\eta Y_{b}=0 ; \\
& \therefore Y_{h}=\frac{r_{h}}{\eta}\left(1-\frac{D_{h}}{K}\right)
\end{aligned}
$$

$$
\check{Z}_{k}=\delta Y_{k}-\left\{d_{k}+(1-f) \frac{r_{k}}{K} D_{k} \mid Z_{h}-0\right.
$$

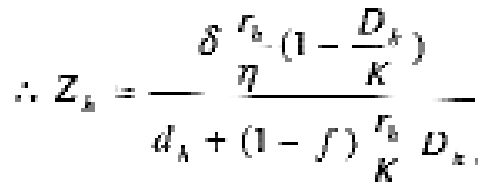

$$
\dot{Y}_{m}=0, \Rightarrow \beta\left(1-f_{s p}\right)\left(D_{m}^{*}-Y_{s}\right) Y_{h}+
$$$$
\gamma\left(1-f_{i p}\right) Z_{h}\left(D_{m}^{*}-Y_{m}\right)-\left(\mu+\mu_{1} D_{m}^{*}\right) Y_{\text {s }}
$$$$
-\kappa_{2} Y_{w}-\kappa_{3} Y_{m}=0
$$

$$
\begin{aligned}
& {\left[\beta^{\prime}+-\frac{\gamma \delta}{d_{q}+(1-f) \frac{r_{A}}{K}} D_{\lambda}\right]}
\end{aligned}
$$

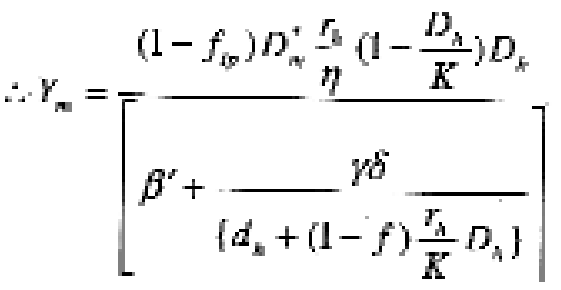

$$
\begin{aligned}
& *\left(1-f_{i p}\right) \frac{r_{h}}{\eta}\left(1-\frac{D_{k}}{K}\right) D_{b}+ \\
& \left(\mu+\kappa_{2}+\kappa_{3}+\mu_{1} D_{m}^{*}\right)
\end{aligned}
$$

Again,

$$
\begin{aligned}
& \beta\left(1-f_{i}\right)\left(D_{k}-Y_{k}-Z_{k}\right) Y_{n}- \\
& \left(v+\eta+d_{b}+\delta+(1-f) \frac{r_{\text {至 }}}{K} D_{h}\right) Y_{b}=0 \\
& \beta\left(1-f_{\phi}\right)\left[1-\frac{r_{h}}{\eta}\left(1-\frac{D_{\mathrm{a}}}{K}\right)\left(1+\frac{\delta}{\left\{d_{\mathrm{A}}+(1-f) \frac{r_{\mathrm{a}}}{K}\right\} D_{\mathrm{a}}}\right] \neq\right.
\end{aligned}
$$$$
D_{k} Y_{n}=\left\{v+\eta+d_{\mathrm{e}}+\delta+(1-f) \frac{T_{h}}{K} D_{\mathrm{h}}\right\} \frac{r_{\mathrm{a}}}{\eta}\left(1-\frac{D_{h}}{K}\right) D_{\mathrm{e}}
$$

From (3.2.9) \& (3.2.10), $D_{\Lambda}=0, D_{h}=K$ or 


$$
\begin{aligned}
& \boldsymbol{F}\left(D_{k}\right)=R_{1} D_{k}^{5}+R_{2} D_{k}^{4}+R_{3} D_{\mathrm{a}}^{3}+R_{4} D_{k}^{2}+ \\
& R_{g} D_{n}+R_{6}=0 \\
& \boldsymbol{R}_{1}=\left\{(1-f) \frac{r_{h}}{K}\right\}^{3} \beta^{\prime}\left(1-f_{T_{T}}\right) \frac{r_{h}}{\eta K} ; \\
& R_{2}=\frac{\beta B D_{\text {m }}}{\eta}(1-f)^{2}\left(1-f_{\psi}\right)^{2}\left(\frac{r_{\mathrm{A}}}{K}\right)^{3}+ \\
& \left\{(1-f) \frac{r_{h}}{K}\right\}^{2}\left\{\left(\beta d_{h}+\gamma \delta\right)\left(1-f_{\varphi}\right) \frac{r_{k}}{\eta K}-\right. \\
& \left.\boldsymbol{\beta}(1-f)\left(1-f_{F}\right) \frac{r_{h}^{2}}{\eta K}\right\}+\beta^{\prime}(1-f)\left(1-f_{\phi}\right) \\
& \left(\frac{r_{\xi}^{2}}{K^{2} \eta}\right)\left\{v+\alpha+d_{h}+\delta(1-f) \frac{r_{h}}{K}+d_{k}(1-f) \frac{r_{\underline{h}}}{K}\right\} . \\
& \boldsymbol{R}_{3}=\frac{\beta\left(1-f_{p}\right)^{2} D_{s}^{*} r_{k}^{2}(1-f)\left(\beta^{\prime} d_{k}+\gamma \delta\right)}{K^{2} \eta}+ \\
& \beta\left(1-f_{\psi_{p}}\right)^{2} D_{\mathrm{m}}^{*} \beta^{\prime}(1-f) \frac{r_{\mathrm{h}}}{K}\left\{\left(1-\frac{r_{h}}{\eta}\right)(1-f) \frac{r_{h}}{K}+\right. \\
& \left.\frac{\boldsymbol{r}_{\boldsymbol{\xi}}}{\boldsymbol{K}}\left(d_{h}+\delta\right)\right\}+\left(v+\eta+d_{h}+\delta\right) \boldsymbol{\beta}^{\prime}(1-f) \frac{r_{k}}{K} * \\
& \left(1-f_{m}\right) \frac{r_{k}}{K \eta}+\left\{\left(\nu+\eta+2 d_{k}+\delta\right)(1-f) \frac{r_{h}}{K}\right\} * \\
& \left\{\left(\beta^{\prime} d_{h}+\gamma \delta\right)\left(1-f_{i p}\right) \frac{r_{k}}{K \eta}-\beta^{\prime}(1-f) *\right. \\
& \left.\left(1-f_{q}\right) \frac{r_{h}^{2}}{K \eta}\right\}-\left\{(1-f) \frac{r_{h}}{K}\right\}^{2}\left\{\mu+\kappa_{2}+\kappa_{3}+\right. \\
& \left.\mu_{1} D_{m}^{*}(1-f) \frac{r_{k}}{K}+\left(\beta^{\prime} d_{b}+\gamma \delta\right)\left(1-f_{m}\right) \frac{r_{k}}{\eta}\right)
\end{aligned}
$$

$$
\begin{aligned}
& R_{4}=\beta\left(1-f_{p}\right)^{2} D_{n}^{+}\left(\beta^{\prime} d_{\mathrm{il}}+\gamma \delta\right)\left\{\left(1-\frac{r_{h}}{\eta}\right) *\right. \\
& \left.(1-f) \frac{r_{h}}{K}+\frac{r_{h}}{\eta K}\left(d_{h}+\delta\right)\right]+\beta\left(1-f_{*}\right)^{2} \text { * } \\
& D_{s}^{+} \beta^{\prime}(1-f) \frac{r_{t}}{K}\left|1-\frac{r_{k}}{K}\left(d_{b}+\delta\right) H(1-f) \frac{r_{k}}{K}\right|^{2} \\
& \left(\mu+\kappa_{2}+\kappa_{j}+\mu_{3} D_{w e}^{*}\right) d_{a}-\left\{\left(v+\eta+2 d_{k}+\delta\right) *\right. \\
& \left.(1-f) \frac{r_{k}}{K}\right\}\left(\mu+\kappa_{2}+\kappa_{3}+\mu_{1} D_{n}^{*}\right)(1-f) \frac{r_{h}}{K}+ \\
& \left.\left(\beta^{\prime} d_{n}+\gamma \delta\right)\left(1-f_{\eta}\right) \frac{r_{k}}{\eta}\right\}+d_{\bar{g}}\left(v+\eta+2 d_{k}+\delta\right) * \\
& \left\{\left(\beta d_{n}+\gamma\right)\left(1-f_{y}\right) \frac{r_{h}}{\eta K}-\beta^{\prime}(1-f)\left(1-f_{w}\right) \frac{r_{b}^{x}}{K^{2} \eta}\right\} . \\
& R_{5}=\beta\left(1-f_{i p}\right)^{2} D_{m}^{4}\left\{1-\frac{r_{h}}{\eta}\left(d_{s}+\delta\right)\right\} \leqslant \\
& \left(\beta^{\prime} d_{h}+\gamma \delta\right)-\mid d d_{h}\left(v+\eta+d_{h}+\delta\right) * \\
& \left\{\left(\mu+\kappa_{i}+\kappa_{3}+\mu_{1} D_{m}^{*}\right)(1-f) \frac{r_{h}}{K}+\right. \\
& \left.\left(\beta d_{k}+\gamma \delta\right)\left(1-f_{p}\right) \frac{r_{k}}{\eta}\right\}+\left(\mu+\kappa_{2}+\right. \\
& \left.\kappa_{3}+\mu_{1} D_{m}^{*}\right) d_{h}\left(\nu+\eta+2 d_{h}+\delta\right)(1-f) \frac{\gamma_{h}}{K} \\
& R_{\mathrm{h}_{1}}=-\left(\begin{array}{l}
\mu+\kappa_{2}+ \\
\kappa_{3}+\mu_{1} D_{m}^{*}
\end{array}\right) d_{h}^{2}\left(\begin{array}{l}
\nu+\eta \eta+ \\
d_{h}+\delta
\end{array}\right) \\
& \text { Now, } F(0)=-\left(\begin{array}{l}
v+\eta+ \\
d_{n}+\delta
\end{array}\right)\left(\begin{array}{l}
\mu+\mu_{1} D_{m}^{*}+ \\
\kappa_{2}+\kappa_{3}
\end{array}\right) d_{h}^{2}<0 \\
& F(K)=\beta\left(1-f_{p}\right)^{2} K D_{m}^{*}\left[d_{\underline{b}}+(1-f) r_{k}\right\} \\
& {\left[\left\{\beta^{\prime} d_{h}+(1-f) r_{h}\right\}+\gamma \delta\right]-\{v+} \\
& \left.\eta+d_{h}+\delta+(1-f) r_{h}\right\}\left(\mu+\mu_{1} D_{\mathrm{nu}}^{*}+\right. \\
& \left.\kappa_{2}+\kappa_{3}\right)\left\{d_{h}+(1-f) r_{h}\right\}^{2}
\end{aligned}
$$

Hence, the sufficient eondition for the existenee of at Icast one positive rool of above (3.2.13) lying between 0 and $\mathrm{K}$ is $F(K)>0$ 


$$
\begin{gathered}
{\left[\beta^{\prime}+\frac{\gamma \delta}{d_{h}+(1-f) r_{\alpha}}\right]} \\
R_{0}[s a y]=\frac{\beta\left(1-f_{b+}\right)^{2} K D_{m}^{*}}{\left\{v+\eta+d_{h}+\delta+(1-f) r_{h}\right\} *}>1 \\
\left(\mu+\mu_{1} D_{m}^{*}+\kappa_{2}+\kappa_{3}\right)
\end{gathered}
$$

This condition $(3.2,14)$ is the threshold condition (which we will see lalter too while showing the instability of disease frec equilibrium, this is important in the scnse that this is the key factor which control the persistence of disease in the pupulution. Knowing, other parameters valucs one can sec what should be the value of $f_{i r}$ and also what should the

value be of $\kappa_{2}$ and $\kappa_{3}$

$$
\text { (when } \kappa_{2} \uparrow, \kappa_{3} \uparrow, D_{\mathrm{s}} \downarrow
$$

$$
\left.\& 2\left(\frac{D_{m}^{+}}{\mu+\mu_{1} D_{m}^{*}+\kappa_{2}+\kappa_{3}}\right) \downarrow\right)
$$

so that

this $R_{v}$ can be made less than one and it will make discasc free cquilihrium to be stable. Here the larval control factor is not coning dircetly into the picture but it is associatod with $D_{\mathrm{w}}^{*}$. The fraction $\frac{D_{\mathrm{w}}^{*}}{\mu+\mu_{1} D_{u}^{*}+\kappa_{2}+\kappa_{3}}$ is increusing with $D_{\mathrm{w}}^{*}$. Hence control $\kappa_{1}$ regulates the total female mosquitoes $D_{\mathrm{m}}^{*}$ and thus affecting this threshold condition. We need to show that there exists only one positive root in the interval 0 and $K$. Here $R_{1}$ is positive and $R_{6}$ is negative, the sufficient condition for $R_{2}$ to be positive is $\left(v+\alpha+2 d_{k}+\delta\right)>r_{h}$. Hence for the existence of unique cquilihrium point, we put following conditions:

$$
\mathrm{R}_{2}>0, \mathrm{R}_{3}>0 \text { and } \mathrm{R}_{\mathrm{s}}<0
$$

So that there will be only one change of sign in (3.2.11). Hence using Descarte's rule of signs, we cun say that there exist at most one pusitive root. After getting this unique pusitive root say $D_{h}^{*}$, it is easy to find $Y_{h}^{*}, Z_{k}^{*}, Y_{m}^{*}$ using oquations $(3.2 .7),(3.2 .8)$ and (3.2.9). Hence the existence of unique cquilibrium point is guaranteed under (3.2.15).

\section{Stability Analysis}

\section{For non trivial equilibrium}

Let, $L=L^{*}+l, Y_{m}=Y_{\mathrm{er}}^{+}+y_{\mathrm{wp},} D_{\mathrm{s}}=D_{\mathrm{au}}^{*}+d_{\mathrm{ru}}$,

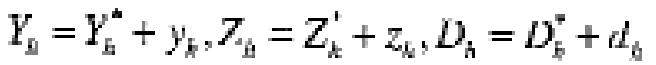

Then the linear system of $(3.2 .1)$ is given by $\frac{d t}{d t}=\frac{d l}{d t}$. Similarly,

$$
\begin{aligned}
& i=b d_{\mathrm{rd}}-\left(c F+\lambda+\kappa_{\mathrm{l}}+2 \lambda_{1} L^{4}\right) l \\
& y_{m}=\left\{\beta^{\prime} y_{k}(t-\tau)+\gamma z_{b}\left(t-\tau_{1}\right)\right\} \\
& \left(1-f_{i q}\right)\left(D_{s}^{*}-Y_{w}^{*}\right)+\left\{\beta Y_{h}^{*}+\gamma^{*} \%_{h}^{*}\right\} \\
& \left(1-f_{i p}\right)\left\{d_{s t}\left(t-\tau_{1}\right)-y_{s w}\left(t-\tau_{1}\right)\right\}- \\
& \left(\mu+\kappa_{2}+\kappa_{\mathrm{3}}+\mu_{1} D_{m}^{*}\right) y_{m}-\mu Y_{\mathrm{M}}^{*} d_{m} \\
& \dot{d}_{a t}=f c F l(t-\tau) \cdots\left(\mu+\kappa_{2}+\kappa_{3}+\right. \\
& \left.2 \mu_{i} D_{w}^{*}\right) d_{a t} \\
& \dot{y}_{b}=\beta\left(1-f_{p}\right)\left[\left(D_{s}^{*}-Y_{b}^{*}-Z_{k}^{*}\right) *\right. \\
& y_{n}\left(t-\tau_{2}\right)+Y_{m}^{*}\left\{d_{\mathrm{a}}\left(t-\tau_{2}\right)-\right. \\
& \left.\left.y_{h}\left(t-\tau_{2}\right)-z_{\hat{f}}\left(t-\tau_{2}\right)\right\}\right]-\{v+ \\
& \left.\eta+d_{N}+\delta+(1-f)_{K}^{r_{h}} D_{h}^{*}\right\} y_{k}- \\
& (1-f){ }_{K}^{r_{k}} \gamma_{\mathrm{a}}^{*} d_{\mathrm{a}}
\end{aligned}
$$

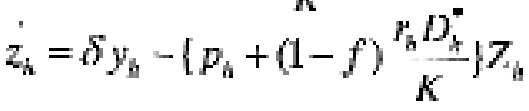

$$
\begin{aligned}
& -(1-f) \frac{r_{k} Z_{k}^{*}}{K} d \text {, } \\
& \dot{d}_{h}=r_{h} d_{h}-\frac{2 r_{h} D_{k}^{*}}{K} d_{\underline{h}}-\eta y_{h}
\end{aligned}
$$

Now louking for the solution in the form of $\sim \exp (\varphi l)$ to get a characteristic polynomial

$\nabla\left(\psi, \tau, \tau_{1}, \tau_{2}\right)=\left\lfloor\psi^{2}+\psi\left(c F+\lambda+\kappa_{1}+\right.\right.$ $\left.2 \lambda_{1} L^{*}+\mu+\kappa_{2}+\kappa_{3}+2 \mu_{1} D_{\mathrm{w}}^{*}\right)+(c F$ $\left.\lambda+\kappa_{1}+2 \lambda_{1} L^{*}\right)\left(\mu+\kappa_{2}+\kappa_{3}+2 \mu_{1} D_{m}^{*}\right)-$ $\left.\alpha b c F e^{-\psi z_{1}}\right] *\left[\psi^{4}+\psi^{3} H_{3}+\psi^{2} H_{2}+\right.$ 


$$
\begin{aligned}
& \boldsymbol{H}_{3}=m_{22}+m_{44}+m_{55}+m_{66} \\
& \left(c F+\lambda+\kappa_{1}+2 \lambda_{2} L^{*}\right) *
\end{aligned}
$$

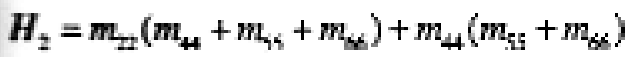

$$
\begin{aligned}
& \left(\mu+\kappa_{2}+\kappa_{3}+2 \mu_{1} D_{m}^{+}\right)>f b c F \\
& +m_{s y} m_{s e}\left[\beta\left(1-f_{q}\right) Y_{m}^{*} e^{v r_{2}}-(1-f) \frac{f_{A}^{*}}{K} Y_{k}^{*} \eta \eta\right. \\
& -\beta\left(1-f_{\varphi}\right)\left(D_{k}^{*}-Y_{k}^{*}-Z_{k}^{*}\right) * \beta^{\prime}\left(1-f_{\psi}\right)\left(D_{k}^{+}-Y_{m}^{*}\right) e^{-\eta_{*}^{*}} \\
& H_{1}=m_{23} m_{41} m_{55}+m_{44} m_{55} m_{66}+ \\
& m_{53} m_{6 s} m_{22}+m_{w s} m_{22} m_{44}+ \\
& \left(m_{22}+m_{s s}\right)\left\{\beta\left(1-f_{p p}\right) * Y_{w 2}^{*} e^{-\phi T_{2}}\right. \\
& \text { and } H_{3}>0,\left|\begin{array}{cc}
H_{3} & H_{1} \\
1 & H_{2}
\end{array}\right|>0 \text {. } \\
& \left|\begin{array}{ccc}
H_{3} & H_{\mathrm{t}} & 0 \\
1 & H_{2} & H_{0} \\
0 & H_{3} & H_{1}
\end{array}\right|>0,\left|\begin{array}{cccc}
H_{1} & H_{1} & 0 & 0 \\
1 & H, & H_{0} & 0 \\
0 & H_{3} & H_{1} & 0 \\
0 & 1 & H_{2} & H_{0}
\end{array}\right|>0
\end{aligned}
$$$$
\left.-(1-f) \frac{r_{h}}{K} Y_{\hat{n}}^{*}\right\} \eta-\beta\left(1-f_{w}\right) Y_{w a}^{*} \eta *
$$$$
(1-f) r_{k} Z_{k}^{*} / K e^{-\psi r_{2}}-\left(m_{\mathrm{ss}}+m_{6 \mathrm{si}}\right) *
$$$$
\beta(1-f)^{2}\left(D_{h}^{*}-Y_{h}^{*}-Z_{k}^{*}\right) \beta^{\prime}\left(D_{m}^{*}-Y_{m}^{*}\right) e^{m_{1}}
$$$$
\boldsymbol{H}_{0}=m_{22} m_{44} m_{33} m_{46}+m_{22} m_{23}\left\{\beta\left(1-f_{p p}\right) *\right.
$$$$
\left.Y_{m}^{*} e^{-r_{2}}-(1-f) \frac{r_{h}}{K} Y_{h}^{*}\right] \eta-m_{22} \beta\left(1-f_{t p}\right) *
$$$$
Y_{m}^{*} e^{-\psi+\gamma /} \eta(1-f) * \frac{r_{h}}{K} Z_{h}^{*}-m_{55} m_{66} \beta \beta^{\prime *} *
$$$$
\left(1-f_{i p}\right)^{2}\left(D_{h}^{*}-Y_{k}^{*}-Z_{k}^{*}\right)
$$$$
m_{2}=\beta^{\prime}\left(1-f_{\nabla}\right) Y_{m}^{*} e^{-v r_{1}}+\gamma\left(1-f_{\nabla}\right) *
$$$$
Z_{h}^{*} e^{-v \pi_{1}}+\mu+\kappa_{2}+\kappa_{3}+\mu_{1} D_{\text {w }}^{+}
$$$$
\boldsymbol{m}_{44}=\beta\left(1-f_{\mathrm{t}}\right) Y_{\mathrm{m}}^{*} e^{\psi \mathrm{rr}_{2}}+v+\eta+
$$$$
p_{k}+\delta+(1-f) \frac{r_{h}}{K} D_{h}^{*}
$$$$
m_{55}=p_{h}+(1-f) \frac{r_{k}}{K} D_{\mathrm{h}^{*}}^{*}
$$$$
m_{66}=\frac{r_{k}}{K}\left(2 D_{\mathrm{h}}^{*}-K\right)\left(D_{n}^{*}-Y_{m}^{*}\right) e^{-m r_{5}}
$$$$
-\beta \gamma\left(1-f_{m}\right)^{2}\left(D_{n}^{*}-Z_{n}^{+}\right)\left(D_{m}^{*}-Y_{m}^{*}\right) *
$$$$
\eta(1-f) \frac{r_{\hat{A}}}{K} Z_{\hat{\hbar}}^{+} e^{-v \tau_{2}}\left(\tau_{1}+\tau_{2}\right)
$$

Here the expression for $\mathrm{H}_{3}, \mathrm{H}_{2}, \mathrm{H}_{1}, \mathrm{H}_{0}$ are siune is defined earlier with $t=t_{1}=\tau_{2}=0$. It is casy to say that the condition (3.3.3) is automatically satisficd lasing fhe eqnation corresponding to the equilibrium of the system (3.2.1). Also the first two inequalities of (3.3.4) are sullislied, so condilions for local asymptotic stahility are the third incquality and $H_{0}>0$. The result of an equilibrium analysis of the model $(3,2,1)$ is stated in the following theorem.

\section{Theorem}

$A$ set of neciessiry und sufficient conditions for $K^{*}\left(I^{*}, Y_{\mathrm{x}}^{*}, I_{s n}^{*}, Y_{\mathrm{b}}^{*}, Z_{\mathrm{b}}^{*}, D_{b}^{*}\right)$ to be asymptotically stable for $t \geq 0, \tau_{1} \geq 0, t_{2} \geq 0$ is the following:

(i) The real parts of all the roots of $\mathrm{V}(a, 0,0,0)=0$ are negative,

(ii) For all real $\omega_{2}$ and $\tau \geq 0, \nabla\left(i \omega d_{2}, \tau, 0,0\right) \neq 0$.

(iii) liur all real $\omega_{2}$ and $\tau_{1} \geq 0, V\left(i \omega_{1}, 0, \tau_{1}, 0\right)+0$,

(iv) For all real $\omega j_{2}$ and $\tau_{2} \geq 0, \mathrm{~V}\left(\mathrm{irs}_{2}, 0,0, \tau_{2}\right) \neq 0$.

Proof: (a) The real parts of all the roots of $\nabla(a, 0,0,0)=0$ are negative, when $\tau=\tau_{1}=z_{2}=0$ then,

$$
\begin{aligned}
& m_{22}=\beta^{\prime}\left(1-f_{\varphi}\right) Y_{m}^{*}+\gamma\left(1-f_{t}\right) Z_{h}^{*}+ \\
& \left(\mu+\kappa_{2}+\kappa_{3}+\mu_{1} D_{m}^{*}\right), \quad m_{44}=\beta\left(1-f_{\varphi}\right) \\
& Y_{m}^{*}+\nu+\eta+p_{h}+\delta+(1 \cdots f) \frac{r_{h}}{K} D_{s}^{*}, \\
& m_{55}=p_{h}+(1-f) \frac{r_{n}}{K} D_{a}, m_{\mathrm{ifb}}=\frac{r_{h}}{K}\left(2 D_{h}^{*}-K\right)
\end{aligned}
$$

For $t=t_{1}=t_{2}=0$, using Routh-Ilurwite criteria the non-trivial (if it cxists) will be locally asymptotically stable if the following condition hold: 
Here we sce $m_{22}, m_{44}, m_{39}$ and $m_{66}$ all are positive. So their multiple additions ure also pusitive. Then the characteristic cquation is

$$
\begin{aligned}
& \nabla(\psi, 0,0,0)=\mid \psi^{2}+\psi\left(c F+\lambda+\kappa_{1}\right. \\
& \left.+2 \lambda_{1} L^{*}+\mu+2 \mu_{1} D_{m}^{*}+\kappa_{2}+\kappa_{3}\right)+ \\
& \left(c F+\lambda+\kappa_{1}+2 \lambda_{1} L^{*}\right)\left(\mu+\kappa_{2}+\kappa_{3}\right. \\
& \left.\left.+2 \mu_{1} D_{m}^{*}\right)-f b c F\right] *\left[\psi^{4}+\psi^{3} H_{3}+\right. \\
& \psi^{2} H_{2}+H_{1} \psi+H_{0} \mid=0 \\
& \left(c F+\lambda+\kappa_{1}+2 \lambda_{1} L^{*}\right) * \\
& \left(\mu+\kappa_{2}+\kappa_{3}+2 \mu_{1} D_{m}^{*}\right)>f b c F
\end{aligned}
$$

and

$$
\begin{aligned}
& H_{4}=\left\{( c F + \lambda + \kappa _ { 1 } + 2 \lambda _ { 1 } L ^ { * } ) \left(\mu+\kappa_{2}+\right.\right. \\
& \left.\left.\kappa_{3}+2 \mu_{1} D_{m}^{*}\right)-f b c F\right\}, \\
& H_{5}=c F+\lambda+\kappa_{1}+2 \lambda_{1} L^{*}+\mu+ \\
& 2 \mu_{1} D_{m}^{*}+\kappa_{2}+\kappa_{3}
\end{aligned}
$$

are also positive. Then

$$
\begin{array}{r}
\nabla(\psi, 0,0,0)=\psi^{6}+\psi^{5}\left(H_{3}+H_{5}\right)+ \\
\psi^{4}\left(H_{2}+H_{5} H_{3}+H_{4}\right)+ \\
\psi^{3}\left(H_{1}+H_{2} H_{5}+H_{4} H_{3}\right)+ \\
\psi^{2}\left(H_{0}+H_{1} H_{5}+H_{2} H_{4}\right)+ \\
\psi\left(H_{5} H_{10}+H_{1} H_{4}\right)+H_{4} H_{0}=0
\end{array}
$$

It is a $6^{\text {th }}$ order polynomial of a function $\psi$. By using Descare's rule of signs there is no pasitive root in this equation which may be negative or imaginary. i.c. some roots are negative and reil. Therefore the real parts of $\nabla(\psi, 0,0,0)=0$ is negutivc, similarly we can say that (b), (c), and (d) is also satisfied then $E^{*}\left(L^{*}, Y_{m}^{*}, D_{m}^{*}, Y_{k}^{*}, Z_{h}^{*}, D_{h}^{*}\right)$ be usymptotically stahle for $\tau \geq 0, z_{1} \geq 0, \tau_{2} \geq 0$. (Proved)

Condition for no stability change of the equilihrium $E^{*}$ due to the delay $t$

In fact in this scetion we are going to see the stability of two dimensional systerns $\left(L-D_{m}\right.$ System) without infection ind how mosquito dynamics uffects the stubility of our system (3.2.1). As $t$ enters only in the firsl factor of the expression $(3.3,2)$ then we get

$$
\begin{aligned}
& \psi^{2}+\left\{c F+\lambda+\kappa_{1}+2 \lambda_{1} L^{*}+\mu+\kappa_{2}+\right. \\
& \kappa_{3}+2 \mu D_{s}^{*} i \psi+\left(c F+\lambda+\kappa_{1}+2 \lambda_{1} L^{*}\right)^{*} \\
& \left(\mu+\kappa_{2}+\kappa_{3}+2 \mu D_{n}^{*}\right)-f b c F_{. e} e^{-v z}=0 \\
& \Rightarrow\left\{\left(\omega_{1}^{2}-\omega_{2}^{2}\right)+\left(c F+\lambda+\kappa_{1}+2 \lambda_{1} L^{*}+\right.\right. \\
& \left.\mu+\kappa_{2}+\kappa_{3}+2 \mu D_{\mathrm{de}}^{*}\right) \omega_{1}-\int h c F e^{\omega_{1} t} * \\
& \cos \omega_{2} \tau+\left(c F+\lambda+\kappa_{1}+2 \lambda_{1} L^{*}\right)(\mu+ \\
& \left.\left.\kappa_{2}+\kappa_{3}+2 \mu D_{m}^{*}\right)\right\}+i\left\{2 \omega _ { 1 } \left(\omega_{2}+\omega_{2}(c F+\right.\right. \\
& \left.\lambda+\kappa_{1}+2 \lambda_{1} L^{\star}+\mu+\kappa_{2}+\kappa_{7}+2 \mu D_{\mathrm{m}}^{\star}\right)+ \\
& \left.f b c F e^{- \text {때 } \tau} \sin \omega_{2} \tau\right\}=0 \\
& {\left[\because \text { Putting } \psi=\omega_{1}+i \omega_{2}\right\rfloor}
\end{aligned}
$$

Now separating real and imaginary part then

$$
\begin{aligned}
& \left\{\left(\omega_{1}^{2}-\omega_{2}^{2}\right)+\left(c F+\lambda+\kappa_{1}+2 \lambda_{1} L^{*}+\mu+\kappa_{2}+\right.\right. \\
& \left.\kappa_{3}+2 \mu D_{m}^{*}\right) \omega_{1}+\left(c F+\lambda+\kappa_{1}+2 \lambda_{2} L^{*}\right) \\
& \left.\left(\mu+\kappa_{2}+\kappa_{3}+2 \mu D_{m}^{*}\right)\right\}-f b c F e^{-v r} \cos \omega_{2} \tau-0 \\
& 2 \omega_{1} \omega_{2}+\omega_{2}\left(c F+\lambda+\kappa_{1}+2 \lambda_{1} I^{*}+\right. \\
& \left.\mu+\kappa_{2}+\kappa_{3}+2 \mu D_{m}^{*}\right)+f b c F e^{-v \sigma \tau} \sin \omega_{2} \tau=0
\end{aligned}
$$

Now putting

$$
A=c F+\lambda+\kappa_{1}+2 \lambda_{1} L^{*} \& B=\mu+\kappa_{2}+\kappa_{3}+2 \mu D_{\mathrm{w}}^{*} ;
$$

then $\omega_{1}=0$

and, $-\omega_{2}{ }^{2}+A B=f b c F \cos \omega_{2} \tau$

$\& \omega_{2}(A+B)=-f b c F \sin \omega_{2} t$

From $(3,4,4) \&(3,4.5)$.

$$
\left(\omega_{2}\right)^{2}+\left(A^{2}+B^{2}\right) \omega_{2}^{2}+A^{2} B^{2}=(j l k: F)^{2}
$$


$\therefore x=\frac{1}{2}\left\{-\left(A^{2}+B^{2}\right) \pm\right.$

$$
\left.\sqrt{\left(A^{4}+B^{4}\right)-2 A^{2} B^{2}+(4 f b c F)^{2}}\right\}
$$

[putting $\omega_{2}=x$ ]

Thus the condition for existence of real $\omega_{2}$ to grel positive $x$ is

$$
\begin{aligned}
& (4 f h c F)^{2}-2 A^{2} B^{2}>0 ; \\
& \therefore f b c F>\frac{1}{\sqrt{2}}(A B)
\end{aligned}
$$

From (3.4.5) \& (3.4.4),

$$
\begin{aligned}
& \tau=\frac{n \pi}{\omega_{2}}+\frac{1}{\omega_{2}} \tan \left(\frac{(A+B) \omega_{2}}{\omega_{2}^{2}-A B}\right) \\
& {\left[\because \text { here } n \geq 0, \beta=\tan ^{-1}\left(\frac{(A+B) \omega_{2}}{\omega_{2}^{2}-A B}\right)\right]:}
\end{aligned}
$$

Hence the necessary and sufficient condition for no stability change is the just opposite condition of (3.4.8).

ie. $f b c F<\frac{1}{\sqrt{2}}(A B)$

\section{Imitation}

Adult female mosquitoes lay c8gs in every 1-2 wecks and lay near about 300 or 10-20 eggs if they have fed or not fed respectively. Consider, an adult female mosquito lays 600 eggs per mouth averagely, larval death tate is taken as $1 / 16$ and the death rate of adult female mosquitoes is $1 / 30$ hecause they survive height 30 days. We dont know the density dependent death rale of mosquilo larvie or idults,

so we takc, $b=20, f=0.5, \quad \lambda=0.0625$, $\lambda_{1}=0.0005, \quad \mu=1 / 30, \quad \mu_{1}=0.0002$. pulting $c F=0.01, \beta=\beta^{2}=0.000002, \gamma=\% / 10$. [For details Burton S.J. \& Cohen, F. (I980), Nedelman, J. (1985) and Ngwa, G.A. \& Shu, W.S. (1999)].

Different areas may have different epidemiological environmenls and it may vary. Suppose a small fraction i.e. $v=0.01$ which corresponds the infections perind of 100 days and $\delta=0.00005$ (fraction going to reservoir class). Wc sssume per capital birth, rate is $b_{h}=30 / 365000 \quad P_{h}=1 / 2190 \mathrm{C}$. The disease related death rate of human is uboul $1.5 \%$ implying per cupita disease induced death rule $7 /=0.015 / 36$. The carrying capucity $K$ is constant. To illustrate the global stability bchavior of $E^{*}$ and to see the eflect of various parameters on the spread of the discasc, the system (3.2.1) is integrated using the fourth order Runge-Kutta method. Initially we tuke all control parameters $K_{1}=0, K_{2}=0, K_{3}=0$, $f_{t p}=0$ and $K-30000$ and delays are $t-20$, $t_{1}=15, \quad t_{x}-15$ 'The equilibrium values ure $\mathrm{L}=6846.95, \mathrm{Y}_{\mathrm{m}}=397.91 \mathrm{I}_{\mathrm{m}}=1227.65$

$$
\mathrm{Y}_{\mathrm{h}}=6202.9 \cdot \mathrm{Z}_{\mathrm{a}}=4836.41 \mathrm{D}_{\mathrm{h}}=18954.7
$$

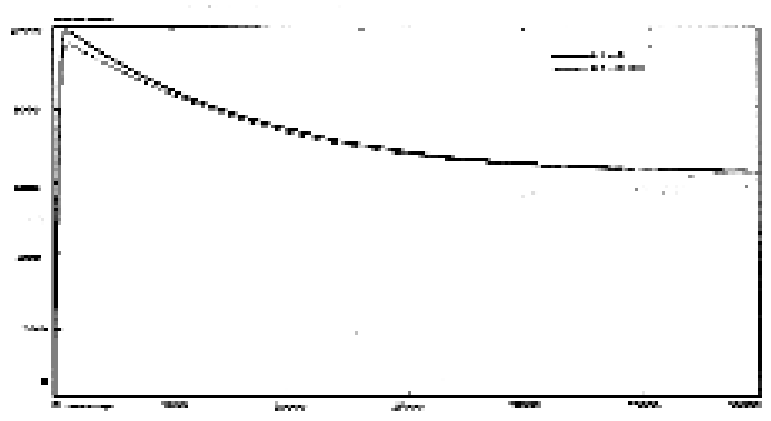

Fig, 4.1: Effect of $k_{l}$ on infocted human.

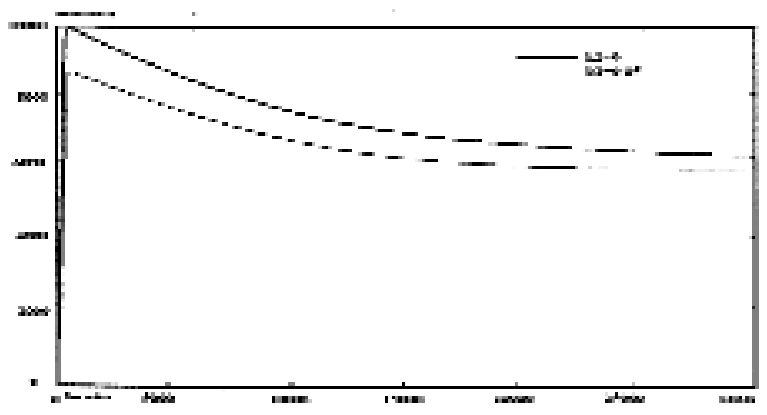

Fig. 4.2: Effect of $k_{2}$ on infected human.

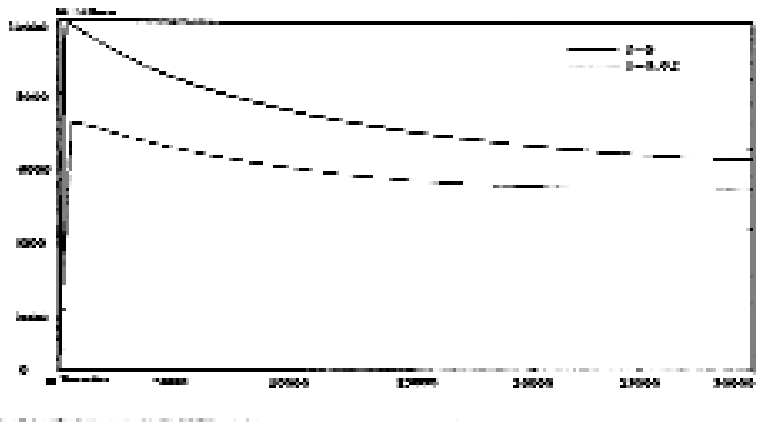

Fig 4,3; Efrect of $\int$ on infected human 


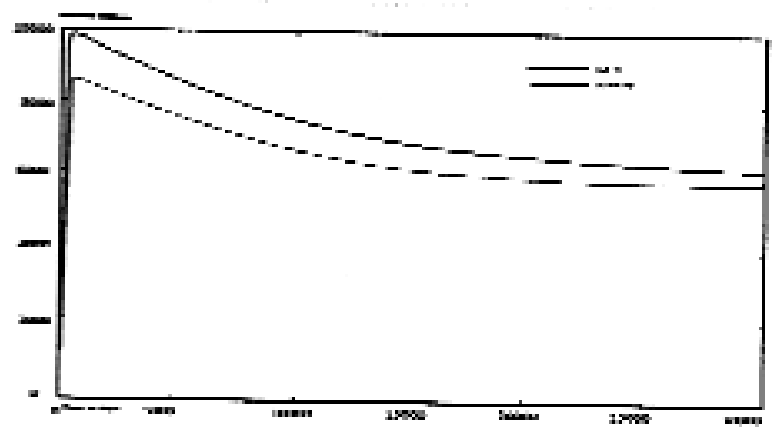

Fig. 4.4: Effect of $k_{3}$ on infected human

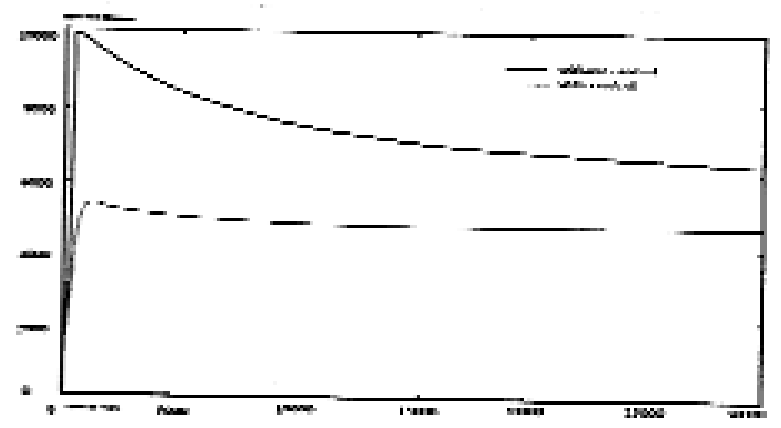

Fig. 4.5: Effect of all contrul measure simultaneously on infected human

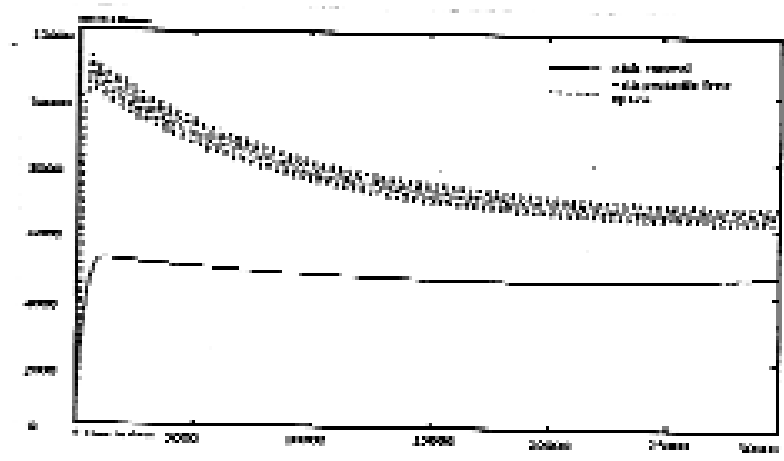

Fig. 4.6: Effect of periodic free space $(F)$ on infected human

Imitation is performed for different initial positions. It is observed that solution curves tend to tile same point for all initial starts implying the global stability of this equilibrium, The effect of different control measures on the infected human population are shown in figures (4.1-4.5). We also consider the cirse in which the frec space $(F)$ is periotic. For example during rainy season water for Bangladesh logging increases frec spice and helps mosquito brocding. It is observod that in this case discase persists with oscillation and with increased infected humans ( $\sec f i g .4 .1-4.6$ ).

\section{Cunclusion}

In this paper a simple SIS model and nonliuear model for malaria transudission in Bangladcsil is proposed and anialyzed. The existence of non trivial equilibrium point is shown under some condition. The conditions for no stability change for the aun trivial equilibrium puint is ohtained. Result on locil asymptotic stability of boundary cquilibrium is also included. Using imitation it is found that the. nontriviul cquilibrium point is in fact globally stahle for the chousen set of parameters. 'The eflects of 'different ontrol mcasures for malaria control ure olbserved and it is concluded that use of bed net is the most effective control meisure for the control of malaria, Parallelism, if we can use other control meisures with bed net then the number of infocted human population decreascs tremendously.

\section{Acknowlcdgement}

The authors wish to thank all honorable teachers of Mathematics Discipline, Khulna University, Khulna, Bangladesh and their parents for providing cssential amenities to fulfill this work.

\section{Keferenccs}

Bailcy N. 1. 1. (1975). The Mathematical theury infectivus diseascs and its applicstions Grif ml. London.

Das P. K, and Amaltaj D. D. (1997). Biolngital control of milaria vecturs. Thaliuen J. Med. Res. 106: 174197.

Dus S, C., Bhuyan M., Baruah L. and Talukder P. K, (1991). Mosquito survey in Tripura, Indiun Jounal of Malariology, 28: PI29 134

Despornmicr D. D., Gwadle R. W. and Hotez. P. J. (11991). Parasitic Discheses 3rd cditive (Springer, Derlin)

Gan, I., Q. and Helhente H. W. (19/2). Disease transmission modcls with deusity dependent demographics, $J$, Math. Bio. 32: P 717-731

Glush, M, (2000) Malthematical Modeling of Inficctious Disease: F.nvironmentill and Dennographic Elfects, Ph.D thesis. (IIT Karapur, India).

Nedelman J. (1985). Introduclory review some new theughts about sone old maliaria models, Math. Biose. 73: 158-182.

Burton S. J. and Cohen F, (1980). Fstimating maliria incidence and rocovery rates from panel surveys, Math. Biosc: 49: 273-305. 
Ghosh, M. und Pugliese, A. (2003). A mathematical Modcling for Malaria in in endemic region: Effect of different control of strategies. NPH, New Delhi, Indiu.

Nchinda T. C. (1998). Malaria: A re-emerging Disease in Africa, Emetging Infoctious Discases. 4(3): 398.

Ngwa, G. A. and Shu, w. S. (1999). A muthematical model for endemic malaria with variable human and mosquito populations. ICTP Preprint No. IC 99158.

Sharma V. P. (1996). Re-emergence of malaria in India, Indian J. Med. Research. 103 : $\mathrm{P} 26-45$,

Sharma V. P. (1998). Fighting malaria in India, Current Science. 75: P 1127-1140.
Walker K. (2002). A review of control methods for African malaria vectors, activity report 108, U.S. Agency for International Development, Washingtun.

WHO (1993, 1995). Vectur cuntrol for malaria and other mosquito-borne discases, WHO (Technical Report Series 857 \& World Malaria Report 2005), Geneval, Switzerland.

Received: June, 18, 2009;

Accepted: March, 22, 2011 